\title{
Study of longitudinal development of cosmic-ray induced air showers with LHAASO-WFCTA
}

\section{Hu Liu ${ }^{a, *}$ on behalf of the LHAASO Collaboration}

(a complete list of authors can be found at the end of the proceedings)

${ }^{a}$ Southwest Jiaotong University,

Xipu Campus, Southwest Jiaotong University, Chengdu, P. R. China, 611756

E-mail: huliu@swjtu.edu.cn

The Wide Field-of-view Cerenkov Telescope Array (WFCTA) is an important component of Large High Altitude Air Shower Observatory (LHAASO), which aims to measure the individual energy spectra of cosmic rays from $\sim 30 \mathrm{TeV}$ to a couple of EeV. The longitudinal development is one of the main tools to distinguish nuclei from each other. WFCTA is composed of 18 imaging air Cerenkov telescopes, each telescope has $32 \times 32$ pixels, covers a field of view $16^{\circ} \times 16^{\circ}$ (each pixel corresponding to $0.5^{\circ} \times 0.5^{\circ}$ ). The first telescope started in operation since February 2019 , up to now, there are 18 telescopes in operation. Cerenkov photon detected by different pixels were generated at different height (or different traversed material). The distribution of $\mathrm{dN} / \mathrm{d} \theta$ ( $\theta$ is the angle between the direction of Cerenkov photon and the direction of primary particle) is reconstructed from the image of WFCTA to study the longitudinal development of the shower ( similar to $\mathrm{dN} / \mathrm{dX}$ ). In this paper, the $\mathrm{dN} / \mathrm{d} \theta$ reconstructing method and the preliminary result on the particle identification power based on this method will be shown.

$37^{\text {th }}$ International Cosmic Ray Conference (ICRC 2021)

July 12 th - 23rd, 2021

Online - Berlin, Germany

\footnotetext{
${ }^{*}$ Presenter
} 


\section{Introduction}

The energy spectra of single element of Cosmic Rays (CRs) in the knee region is the key to understand the physics of knee [1]. Currently the energy spectrum in this energy region are only measured by the ground experiment. For ground experiment, the longitudinal development of the shower (or $\mathrm{dN} / \mathrm{dX}[2]$ ) is one of the main tools to distinguish nuclei from each other. LHAASO located in Haizishan Daocheng, Sichuan province of China, is a hybrid experiment to detect cosmic rays. The height of LHAASO is $4410 \mathrm{~m}$ above sea level. One of the main scientific goals is measuring the energy spectra of individual element from $\sim 30 \mathrm{TeV}$ to several EeV. LHAASO has 18 Wide Field-of-view Cerenkov Telescopes, which are used to detect the image of Cerenkov/fluorescent radiation of Extensive Air Shower (EAS) induced by cosmic rays. LHAASO also has 78,000 $\mathrm{m}^{2}$ water Cerenkov detector (WCDA), and $1 \mathrm{~km}^{2}$ Array (KM2A). Both WCDA and KM2A will provide the core position and direction measurement. By combining the image measurement from WFCTA and the core position and direction measurement from KM2A, the number of Cerenkov photons in different $\theta$ bins (noted as $\mathrm{dN} / \mathrm{d} \theta, \theta$ is the angle between the direction of Cerenkov photon and the direction of primary particle) is reconstructed both for data and Monte Carlo (MC) samples to study the longitudinal development of the shower induced by cosmic rays. The reconstructing method and preliminary result on particle identification will be shown in this paper.

\section{Experiment}

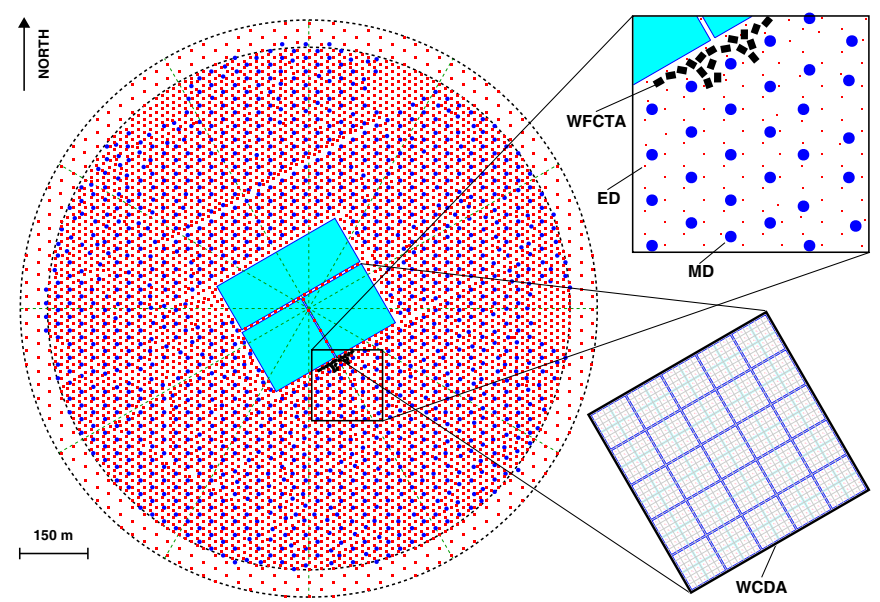

Figure 1: The layout of LHAASO experiment, WFCTA located at the center of KM2A array.

The layout of LHAASO is shown in Figure 1. WFCTA located at the center of the KM2A array, close to the edges of WCDA. Both KM2A and WCDA will provide the core position with a few meters resolution and the direction of the primary particle with resolution less than $0.5^{\circ}$ [3]. WFCTA detect the image of Cerenkov radiation from the EAS. Each Cerenkov telescope of WFCTA consists of an array of $32 \times 32$ Silicon Photo Multipliers (SiPMs) and a $\sim 5 \mathrm{~m}^{2}$ spherical aluminised mirror. It has a field of view $16^{\circ} \times 16^{\circ}$ with a pixel size of approximately $0.5^{\circ} \times$ $0.5^{\circ}$. The telescope unit together with the power supply and a slow control system are installed 
in a $4.2 \mathrm{~m} \times 2.5 \mathrm{~m} \times 2.6 \mathrm{~m}$ shipping container. Different configurations of the telescopes in the array will be used to optimise the sensitivity to the measurement of the cosmic ray energy spectrum and composition in different energy ranges. In the first phase, there are six telescopes, the field of view of the 6 telescopes are shown in Figure 2 (noted as T1,...,T6 below), there are overlapped area between telescopes to reduce the leakage of the image. KM2A is composed of 5195 electromagnetic detectors (ED) and 1188 muon detectors (MD). The direction of primary particle is reconstructed by fitting the relative arriving time of ED hits, the core position is reconstructed by fitting the lateral distribution of secondary charged particles detected by EDs. The event of WFCTA and KM2A are matched together by selecting the difference of the trigger time within a few microsecond. The analysis in this paper is based on the data collected by WFCTA and KM2A during the first phase.

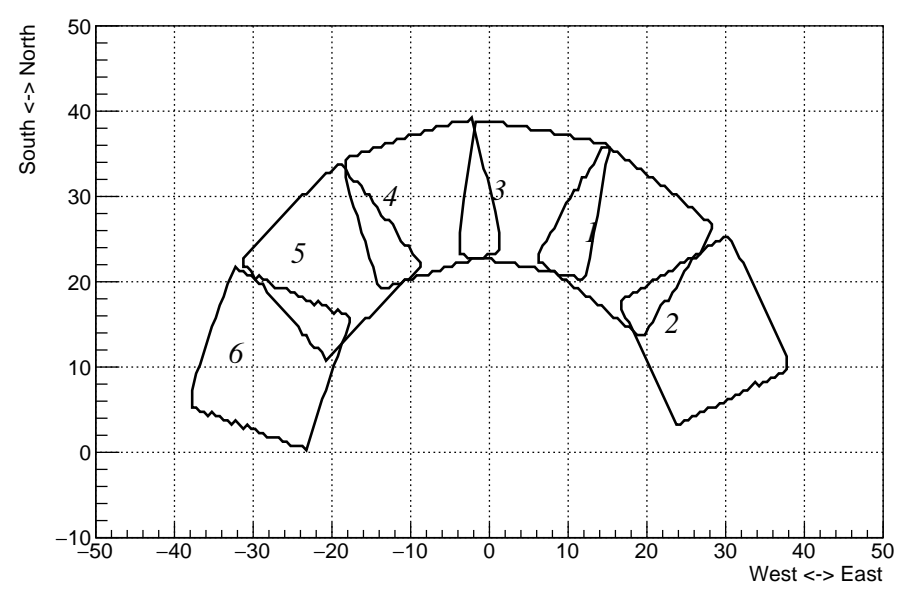

Figure 2: The field of view of 6 telescopes of WFCTA

\section{Monte Carlo simulation}

The simulations performed include the detailed air shower development in the atmosphere, as well as the response of the detectors of KM2A and WFCTA. Air showers were simulated in a wide energy range from $10^{13}$ to $10^{16} \mathrm{eV}$ with CORSIKA (v76400) [4]. The QGSJETII models for high-energy and FLUKA for low-energy hadronic interactions were used, and the EGS4 model was used for electromagnetic interactions. Five components-protons, helium, $\mathrm{CNO}, \mathrm{MgAlSi}$, and iron are generated in the above mentioned energy range, the mass number (noted as A below) for the $\mathrm{CNO}$ and MgAlSi groups is 14 and 27, respectively. Showers following an $E^{-1}$ energy spectrum and an isotropic angular distribution are simulated with a zenith angle range of 20-40 degrees and an azimuth range of 0-180 degrees, to match WFCTA field of view. The secondary particles and Cerenkov photons reaching ground level are treated in a delicately developed detector simulation program. The program for KM2A is developed in the framework of Geant4 package [5], The program for WFCTA is developed based on ray tracing method. The shower core position is evenly distributed in a square with the center of the WFCTA as the origin and a length of 600 meters. 


\section{The Method}

A typical image of EAS detected by one telescope of WFCTA is shown in the left panel of Figure 3. The longitudinal development of the shower can be seen from the change of number of Cerenkov photons along the long axis of the image, however, the image is also affected by the viewing angle of the telescope. Every shower event has different direction, to study the longitudinal development with the same view angle. A virtual image from a virtual telescope is reconstructed. The direction of the virtual telescope is parallel with direction of the primary particle. The direction
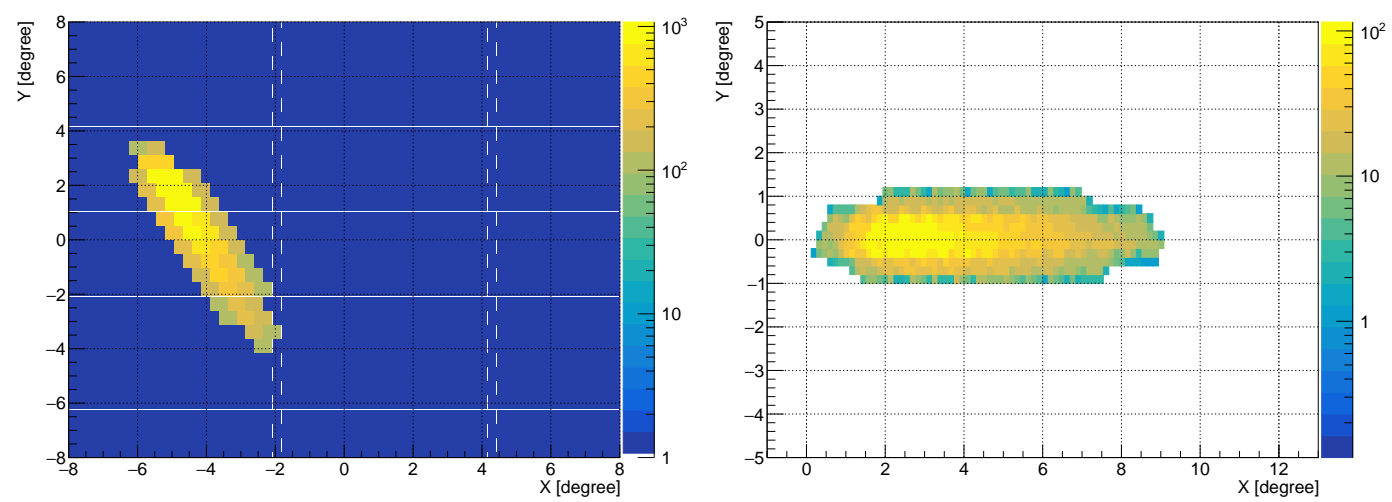

Figure 3: left: The image of Cerenkov photons detected by T1 of WFCTA; right: the image of the same event calculated for the virtual telescope, which points to the direction of the primary particle.

of the virtual telescope is set to the reconstructed direction of the primary particle (defined as xaxis), while the position of the telescope stays the same. The $x$-axis of the virtual telescope is perpendicular to the z-axis, and points to the direction of the core position. The y-axis is the cross product of z-axis and x-axis. For the signal of each SiPM from the real telescope, the direction of those photons are calculated. Also the efficiency is used to calculate the number of photons before entering the telescope, and then the detector simulation of the virtual telescope is performed for those photons. Most of the efficiencies used in previous two steps are the same, the impact of the inaccuracy of efficiency is cancelled out. After those procedures, the image from the virtual telescope is obtained, which is shown in the right panel of Figure 3.

There are also some events detected by two telescopes, a typical event is shown in Figure 4. In Figure 4(a), part of the event is detected by T1 (left) and T2 (right). After the same calculation procedures with the event detected by one telescope, the image from the virtual telescope is shown in Figure 4(b), the left one is the image from T1, the middle one is the image from T2, and the right one is the combined image from $\mathrm{T} 1$ and $\mathrm{T} 2$. For the overlapped bins between $\mathrm{T} 1$ and $\mathrm{T} 2$, an average value is used for the combined image.

The projection of the combined image of the virtual telescope to the $\mathrm{x}$-axis is shown in Figure 5 , the left one is the event detected by single telescope, and the right one is the event detected by two telescopes. This distribution is defined as $\mathrm{dN} / d \theta$, where $\theta$ is the angle between the direction of Cerenkov photons and the direction of primary particle, $\mathrm{N}$ is the number of Cerenkov photons in the $\theta$ bin. Normally the longitudinal development function is refereed to $\mathrm{dN} / \mathrm{dX}$, where $\mathrm{X}$ is the 

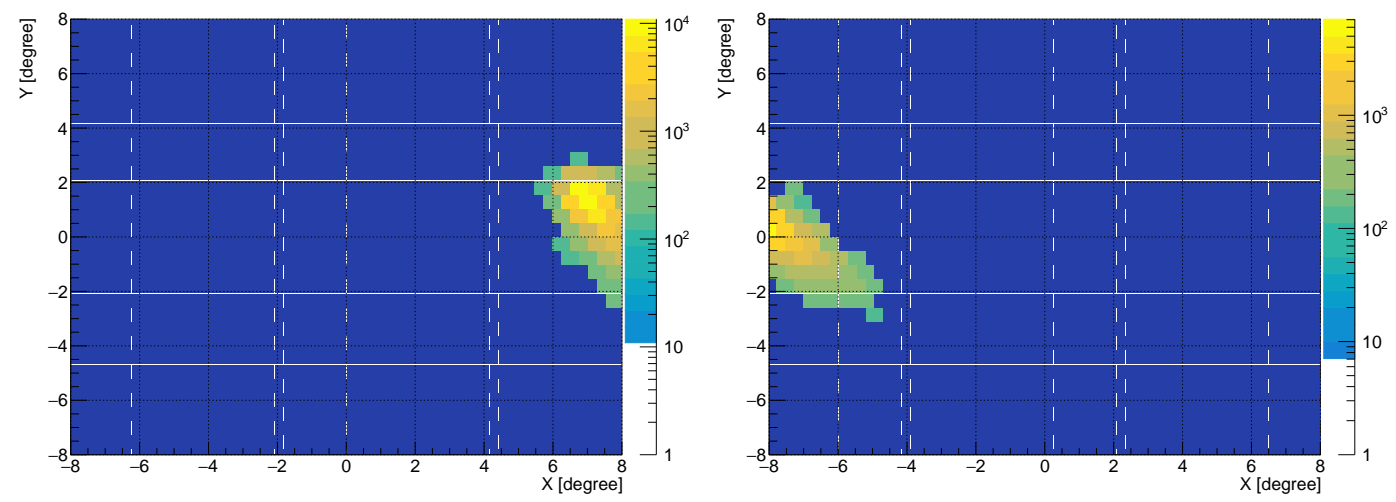

(a)
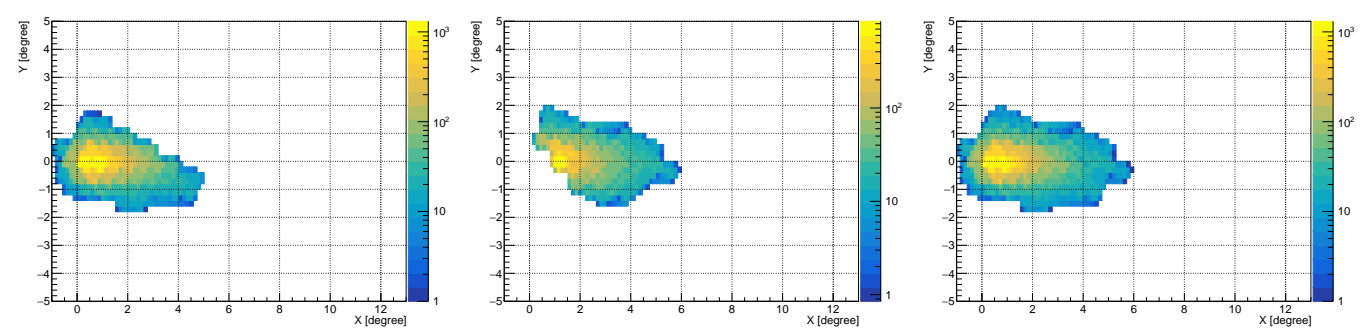

(b)

Figure 4: (a): The image of Cerenkov photons detected by T1 (left) and T2 (right) of WFCTA; (b): The image of the same event calculated for the virtual telescope, which points to the direction of the primary particle. Left: T1; middle: T2; right: T1 and T2 combined.

atmosphere mass traversed. In principle, the $\theta$ angle is correlated with X, because different $\theta$ angle corresponding to different Cerenkov photon production height. However, based on MC simulation, it can be seen that the relationship between $\theta$ and $\mathrm{X}$ is also affected by other variables, for example, the mass of the primary particle. So in this paper, the new distribution of $\mathrm{dN} / d \theta$ is used to study the longitudinal development of the shower, instead of $\mathrm{dN} / \mathrm{dX}$.

From MC simulation, The small $\theta$ region of $\mathrm{dN} / d \theta$ is well fitted with a Gaussian function, while the large $\theta$ region of $\mathrm{dN} / d \theta$ decreases slower than an exponential function, and is well fitted with the function of $e^{-k \theta^{\alpha}}$. In this paper, the convolution of Gaussian function and this function is reconstructed to fit the data of $\mathrm{dN} / d \theta$. The formula of the function is equation 1 , the fitting result for the same two events is shown in Figure 5. The key parameter from 1 is the $\theta$ at which the $\mathrm{dN} / d \theta$ reaches maximum (noted as $\theta_{\max }$ below), $\theta_{\max }$ will be used to do the particle identification between different nuclei.

$$
f(\theta)=N e^{-\frac{(\theta-\mu)^{2}}{2 \sigma^{2}}} \otimes e^{-k \theta^{\alpha}}
$$



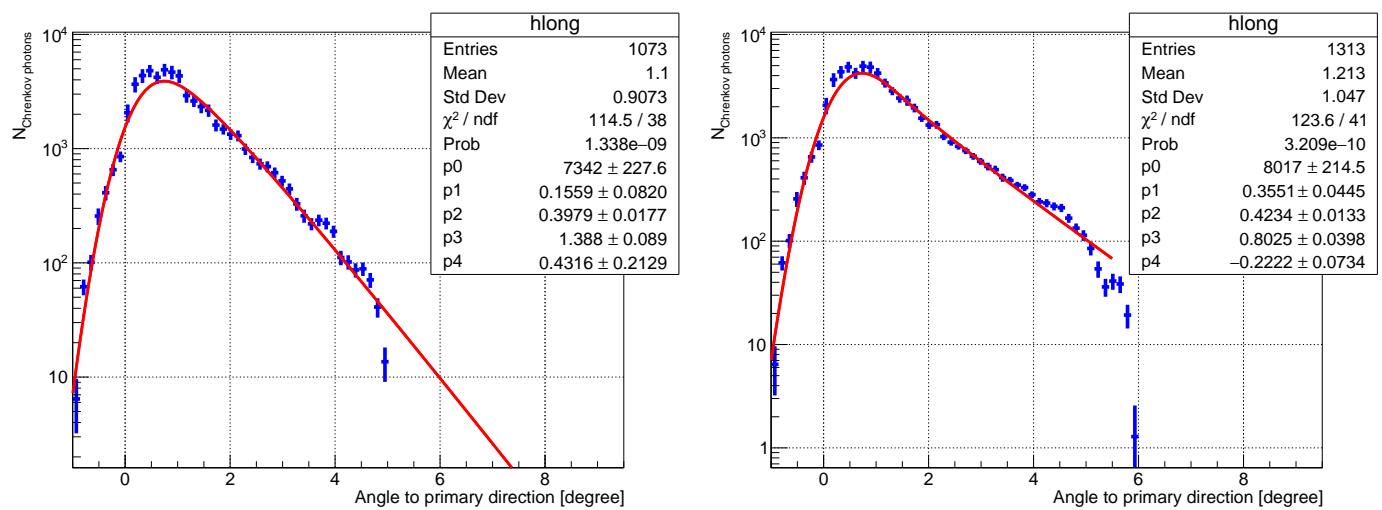

Figure 5: The distribution of $\mathrm{dN} / \mathrm{d} \theta$ for two events of cosmic ray data, red line is the fitted function of equation 1. Left: the event fully detected by single telescope; right: the event detected by two telescopes, and the images are combined together.

\section{Result}

To study the particle identification power of $\theta_{\max }$, the same procedures are performed for the MC samples. The distribution of $\mathrm{dN} / d \theta$ from MC samples is similar to the one from the data, and can also be well fitted with equation 1 . From MC samples, $\theta_{\max }$ is mostly affected by energy and mass of the primary particle, however, it is also linear correlated with the Rp parameter, which is the perpendicular distance from the telescope to the shower axis. After correcting for the Rp effect, a preliminary result of the distribution of $\theta_{\max }$ for proton and iron MC samples at around $1 \mathrm{PeV}$ is shown in Figure 6.

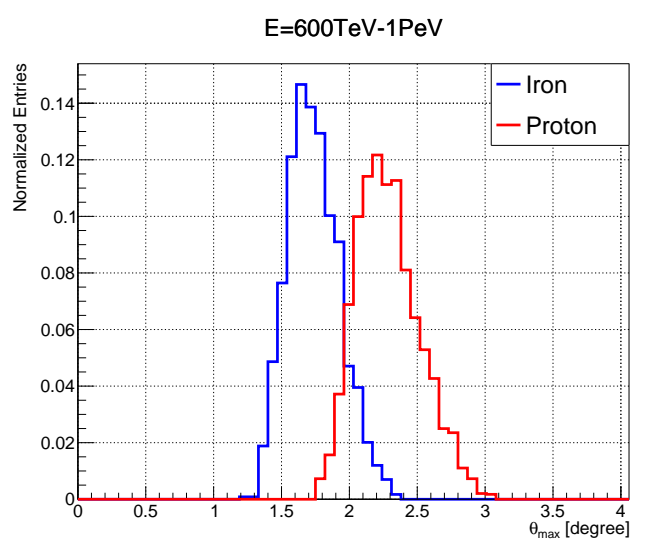

Figure 6: Preliminary result on the distribution of $\theta_{\max }$ for proton (red) and iron (blue) MC samples in the energy range from $600 \mathrm{TeV}$ to $1 \mathrm{PeV}$.

\section{Acknowledgements}

This work is supported in China by the Fundamental Research Funds for the Central Universities (grant numbers 2682020CX77, 2682020CX73, 2682020CX74). It is also supported by 
the Science and Technology Department of Sichuan Province (grant numbers 2021YFSY0031, 2020YFSY0016), and by NSFC (grant number 11947404), and by National Key R\&D program of China (grant number 2018YFA0404201).

\section{References}

[1] B. Bartoli et al., PHYSICAL REVIEW D 92, 092005 (2015)

[2] T. K. Gaisser, A. M. Hillas, Proc. 15th ICRC 8, 353 (1977)

[3] F. Aharonian et al., Chin. Phys. C 45, 025002 (2021)

[4] D. Heck et al., CORSIKA: a Monte Carlo code to simulate extensive air showers. (1998)

[5] S. Agostinelli et al., Nucl. Instrum. Methods Phys. Res., Sect. A 506, 250 (2003) 


\section{Full Authors List: LHAASO Collaboration}

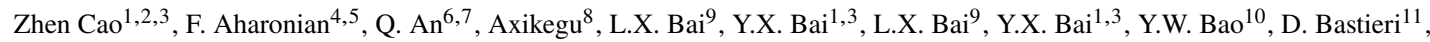
X.J. Bi ${ }^{1,2,3}$, Y.J. Bi ${ }^{1,3}$, H. Cai ${ }^{12}$, J.T. Cai ${ }^{11}$, Zhe Cao ${ }^{6,7}$, J. Chang ${ }^{13}$, J.F. Chang ${ }^{1,3,6}$, B.M. Chen ${ }^{14}$, E.S. Chen ${ }^{1,2,3}$, J. Chen ${ }^{9}$, Liang Chen $^{1,2,3}$, Liang Chen ${ }^{15}$, Long Chen ${ }^{8}$, M.J. Chen ${ }^{1,3}$, M.L. Chen ${ }^{1,3,6}$, Q.H. Chen ${ }^{8}$, S.H. Chen ${ }^{1,2,3}$, S.Z. Chen ${ }^{1,3}$, T.L. Chen ${ }^{16}$,X.L. $\mathrm{Chen}^{1,2,3}$, Y. Chen $^{10}$, N. Cheng ${ }^{1,3}$, Y.D. Cheng ${ }^{1,3}$, S.W. Cui ${ }^{14}$, X.H. Cuii ${ }^{17}$, Y.D. Cui ${ }^{18}$, B. D'Ettorre Piazzoli ${ }^{19}$, B.Z. Dai ${ }^{20}$, H.L.

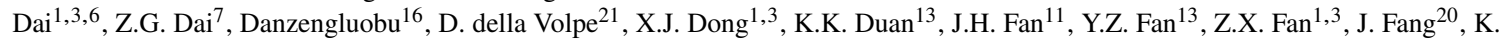
Fang $^{1,3}$, C.F. Feng ${ }^{22}$, L. Feng ${ }^{13}$, S.H. Feng ${ }^{1,3}$, Y.L. Feng ${ }^{13}$, B. Gao ${ }^{1,3}$, C.D. Gao ${ }^{22}$, L.Q. Gao ${ }^{1,2,3}$, Q. Gao ${ }^{16}$, W. Gao ${ }^{22}$, M.M. Ge ${ }^{20}$, L.S. Geng ${ }^{1,3}$, G.H. Gong ${ }^{23}$, Q.B. Gou ${ }^{1,3}$, M.H. Gu${ }^{1,3,6}$, F.L. Guo ${ }^{15}$, J.G. Guo ${ }^{1,2,3}$, X.L. Guo ${ }^{8}$, Y.Q. Guo ${ }^{1,3}$, Y.Y. Guo ${ }^{1,2,3,13}$, Y.A. $\mathrm{Han}^{24}$, H.H. He $\mathrm{H}^{1,2,3}$, H.N. He ${ }^{13}$, J.C. He ${ }^{1,2,3}$, S.L. He ${ }^{11}$, X.B. He ${ }^{18}$, Y. He ${ }^{8}$, M. Heller ${ }^{21}$, Y.K. Hor ${ }^{18}$, C. Hou ${ }^{1,3}$, H.B. Hu ${ }^{1,2,3}$, S.

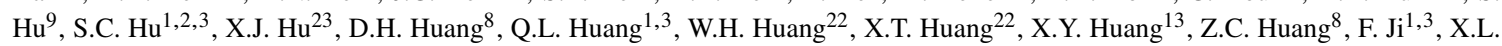
$\mathrm{Ji}^{1,3,6}$, H.Y. Jia ${ }^{8}$, K. Jiang ${ }^{6,7}$, Z.J. Jiang ${ }^{20}$, C. Jin ${ }^{1,2,3}$, T. Ke ${ }^{1,3}$, D. Kuleshov ${ }^{25}$, K. Levochkin ${ }^{25}$, B.B. Li ${ }^{14}$, Cheng Li ${ }^{6,7}$, Cong Li ${ }^{1,3}$,

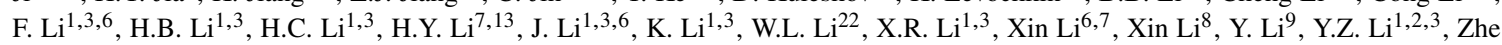
$\mathrm{Li}^{1,3}$, Zhuo Li ${ }^{26}$, E.W. Liang ${ }^{27}$, Y.F. Liang ${ }^{27}$, S.J. Lin ${ }^{18}$, B. Liu ${ }^{7}$, C. Liu ${ }^{1,3}$, D. Liu ${ }^{22}$, H. Liu ${ }^{8}$, H.D. Liu ${ }^{24}$, J. Liu ${ }^{1,3}$, J.L. Liu ${ }^{28}$, J.S.

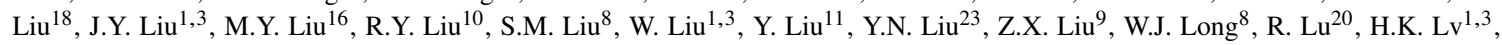

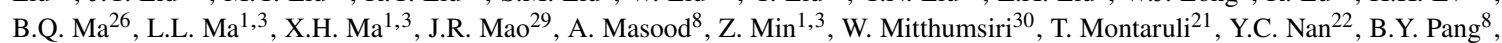
P. Pattarakijwanich ${ }^{30}$, Z.Y. Pei ${ }^{11}$, M.Y. Qi ${ }^{1,3}$, Y.Q. Qi ${ }^{14}$, B.Q. Qiao ${ }^{1,3}$, J.J. Qin ${ }^{7}$, D. Ruffolo ${ }^{30}$, V. Rulev ${ }^{25}$, A. Sáiz ${ }^{30}$, L. Shao ${ }^{14}$, O. Shchegolev $^{25,31}$, X.D. Sheng ${ }^{1,3}$, J.Y. Shi ${ }^{1,3}$, H.C. Song ${ }^{26}$, Yu.V. Stenkin ${ }^{25,31}$, V. Stepanov ${ }^{25}$, Y. Su ${ }^{32}$, Q.N. Sun ${ }^{8}$, X.N. Sun ${ }^{27}$, Z.B.

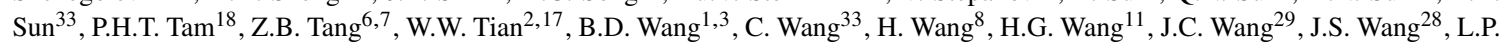
Wang $^{22}$, L.Y. Wang ${ }^{1,3}$, R.N. Wang ${ }^{8}$, W. Wang ${ }^{18}$, W. Wang ${ }^{12}$, X.G. Wang ${ }^{27}$, X.J. Wang ${ }^{1,3}$, X.Y. Wang ${ }^{10}$, Y. Wang ${ }^{8}$, Y.D. Wang ${ }^{1,3}$, Y.J. Wang $^{1,3}$, Y.P. Wang ${ }^{1,2,3}$, Z.H. Wang ${ }^{9}$, Z.X. Wang ${ }^{20}$, Zhen Wang ${ }^{28}$, Zheng Wang ${ }^{1,3,6}$, D.M. Wei ${ }^{13}$, J.J. Wei ${ }^{13}$, Y.J. Wei ${ }^{1,2,3}$, T. Wen $^{20}$, C.Y. Wu ${ }^{1,3}$, H.R. Wu ${ }^{1,3}$, S. $\mathrm{Wu}^{1,3}$, W.X. Wu ${ }^{8}$, X.F. Wu ${ }^{13}$, S.Q. Xi ${ }^{1,3}$, J. Xia ${ }^{7}, 13$, J.J. Xia ${ }^{8}$, G.M. Xiang ${ }^{2,15}$, D.X. Xiao ${ }^{16}$, G. Xiao ${ }^{1,3}$, H.B. Xiao ${ }^{11}$, G.G. Xin ${ }^{12}$, Y.L. Xin ${ }^{8}$, Y. Xing ${ }^{15}$, D.L. Xu ${ }^{28}$, R.X. Xu ${ }^{26}$, L. Xue ${ }^{22}$, D.H. Yan ${ }^{29}$, J.Z. Yan ${ }^{13}$, C.W. Yang 9 , F.F. Yang ${ }^{1,3,6}$, J.Y. Yang ${ }^{18}$, L.L. Yang ${ }^{18}$, M.J. Yang ${ }^{1,3}$, R.Z. Yang ${ }^{7}$, S.B. Yang ${ }^{20}$, Y.H. Yao ${ }^{9}$, Z.G. Yao ${ }^{1,3}$, Y.M. Ye ${ }^{23}$, L.Q. Yin ${ }^{1,3}$, N. Yin ${ }^{22}$, X.H. You $^{1,3}$, Z.Y. You ${ }^{1,2,3}$, Y.H. Yu ${ }^{22}$, Q. Yuan ${ }^{13}$, H.D. Zeng ${ }^{13}$, T.X. Zeng ${ }^{1,3,6}$, W. Zeng ${ }^{20}$, Z.K. Zeng ${ }^{1,2,3}$, M. Zha ${ }^{1,3}$, X.X. Zhai ${ }^{1,3}$, B.B. Zhang $^{10}$, H.M. Zhang ${ }^{10}$, H.Y. Zhang ${ }^{22}$, J.L. Zhang ${ }^{17}$, J.W. Zhang ${ }^{9}$, L.X. Zhang ${ }^{11}$, Li Zhang ${ }^{20}$, Lu Zhang ${ }^{14}$, P.F. Zhang ${ }^{20}$, P.P. Zhang ${ }^{14}$, R. Zhang ${ }^{7,13}$, S.R. Zhang ${ }^{14}$, S.S. Zhang ${ }^{1,3}$, X. Zhang ${ }^{10}$, X.P. Zhang ${ }^{1,3}$, Y.F. Zhang ${ }^{8}$, Y.L. Zhang ${ }^{1,3}$, Yi Zhang ${ }^{1,13}$, Yong Zhang ${ }^{1,3}$, B. $Z_{\text {Zhao }}^{8}$, J. Zhao ${ }^{1,3}$, L. Zhao ${ }^{6,7}$, L.Z. Zhao ${ }^{14}$, S.P. Zhao ${ }^{13,22}$, F. Zheng ${ }^{33}$, Y. Zheng ${ }^{8}$, B. Zhou ${ }^{1,3}$, H. Zhou ${ }^{28}$, J.N. Zhou ${ }^{15}$, P. Zhou ${ }^{10}$, R. Zhou $^{9}$, X.X. Zhou ${ }^{8}$, C.G. Zhu ${ }^{22}$, F.R. Zhu ${ }^{8}$, H. Zhu ${ }^{17}$, K.J. Zhu ${ }^{1,2,3,6}$ and X. Zuo ${ }^{1,3}$

${ }^{1}$ Key Laboratory of Particle Astrophyics \& Experimental Physics Division \& Computing Center, Institute of High Energy Physics, Chinese Academy of Sciences, 100049 Beijing, China.

${ }^{2}$ University of Chinese Academy of Sciences, 100049 Beijing, China.

${ }^{3}$ TIANFU Cosmic Ray Research Center, Chengdu, Sichuan, China.

${ }^{4}$ Dublin Institute for Advanced Studies, 31 Fitzwilliam Place, 2 Dublin, Ireland.

${ }^{5}$ Max-Planck-Institut for Nuclear Physics, P.O. Box 103980, 69029 Heidelberg, Germany.

${ }^{6}$ State Key Laboratory of Particle Detection and Electronics, China.

${ }^{7}$ University of Science and Technology of China, 230026 Hefei, Anhui, China.

${ }^{8}$ School of Physical Science and Technology \& School of Information Science and Technology, Southwest Jiaotong University, 610031 Chengdu, Sichuan, China.

${ }^{9}$ College of Physics, Sichuan University, 610065 Chengdu, Sichuan, China.

${ }^{10}$ School of Astronomy and Space Science, Nanjing University, 210023 Nanjing, Jiangsu, China.

${ }^{11}$ Center for Astrophysics, Guangzhou University, 510006 Guangzhou, Guangdong, China.

${ }^{12}$ School of Physics and Technology, Wuhan University, 430072 Wuhan, Hubei, China.

${ }^{13}$ Key Laboratory of Dark Matter and Space Astronomy, Purple Mountain Observatory, Chinese Academy of Sciences, 210023 Nanjing, Jiangsu, China.

${ }^{14}$ Hebei Normal University, 050024 Shijiazhuang, Hebei, China.

${ }^{15}$ Key Laboratory for Research in Galaxies and Cosmology, Shanghai Astronomical Observatory, Chinese Academy of Sciences, 200030 Shanghai, China.

${ }^{16}$ Key Laboratory of Cosmic Rays (Tibet University), Ministry of Education, 850000 Lhasa, Tibet, China.

${ }^{17}$ National Astronomical Observatories, Chinese Academy of Sciences, 100101 Beijing, China.

${ }^{18}$ School of Physics and Astronomy \& School of Physics (Guangzhou), Sun Yat-sen University, 519000 Zhuhai, Guangdong, China.

${ }^{19}$ Dipartimento di Fisica dell'Università di Napoli 'Federico II', Complesso Universitario di Monte Sant’Angelo, via Cinthia, 80126 Napoli, Italy.

${ }^{20}$ School of Physics and Astronomy, Yunnan University, 650091 Kunming, Yunnan, China.

${ }^{21} \mathrm{D}_{i}{ }^{-}$epartement de Physique Nucl ${ }_{i}{ }^{-}$eaire et Corpusculaire, Facult ${ }_{i}^{-}$e de Sciences, Universiti ${ }^{-}$e de Gen 'eve, 24 Quai Ernest Ansermet, 1211 Geneva, Switzerland.

${ }^{22}$ Institute of Frontier and Interdisciplinary Science, Shandong University, 266237 Qingdao, Shandong, China.

${ }^{23}$ Department of Engineering Physics, Tsinghua University, 100084 Beijing, China.

${ }^{24}$ School of Physics and Microelectronics, Zhengzhou University, 450001 Zhengzhou, Henan, China.

${ }^{25}$ Institute for Nuclear Research of Russian Academy of Sciences, 117312 Moscow, Russia. 
${ }^{26}$ School of Physics, Peking University, 100871 Beijing, China.

${ }^{27}$ School of Physical Science and Technology, Guangxi University, 530004 Nanning, Guangxi, China.

${ }^{28}$ Tsung-Dao Lee Institute \& School of Physics and Astronomy, Shanghai Jiao Tong University, 200240 Shanghai, China.

${ }^{29}$ Yunnan Observatories, Chinese Academy of Sciences, 650216 Kunming, Yunnan, China.

${ }^{30}$ Department of Physics, Faculty of Science, Mahidol University, 10400 Bangkok, Thailand.

${ }^{31}$ Moscow Institute of Physics and Technology, 141700 Moscow, Russia.

${ }^{32}$ Key Laboratory of Radio Astronomy, Purple Mountain Observatory, Chinese Academy of Sciences, 210023 Nanjing, Jiangsu, China.

${ }^{33}$ National Space Science Center, Chinese Academy of Sciences, 100190 Beijing, China. 